\title{
Modelagem matemática aplicada a cinética de secagem da pasta de amido de feijão preto
}

\author{
Mathematical modeling applied to the drying kinetics of black bean starch paste \\ Modelado matemático aplicado a la cinética de secado de la pasta de almidón de frijol negro
}

Recebido: 12/01/2021 | Revisado: 15/01/2021 | Aceito: 15/01/2021 | Publicado: 18/01/2021

Raphael Lucas Jacinto Almeida

ORCID: https://orcid.org/0000-0001-7232-2373 Universidade Federal do Rio Grande do Norte, Brasil E-mail: raphaelqindustrial@gmail.com

Newton Carlos Santos

ORCID: https://orcid.org/0000-0002-9603-2503 Universidade Federal do Rio Grande do Norte, Brasil

E-mail: newtonquimicoindustrial@gmail.com

Victor Herbert de Alcântara Ribeiro

ORCID: https://orcid.org/0000-0001-6286-5403

Universidade Federal de Campina Grande, Brasil E-mail:victor_herbert@hotmail.com

Virgínia Mirtes de Alcântara Silva ORCID: https://orcid.org/0000-0001-6493-3203

Universidade Federal de Campina Grande, Brasil E-mail:virginia.mirtes2015@gmail.com

Cecília Elisa de Sousa Muniz ORCID: https://orcid.org/0000-0002-7402-9354 Universidade Federal de Campina Grande, Brasil E-mail:ceciliamuniz.qi@gmail.com

Rebeca de Almeida Silva

ORCID: https://orcid.org/0000-0002-4116-5050

Centro Universitário Maurício de Nassau, Brasil E-mail:rebecaalmeidasilva@gmail.com

Raphael da Silva Eduardo

ORCID: https://orcid.org/0000-0002-5968-7578

Universidade Federal de Campina Grande, Brasil

E-mail:raphael_leahpar17@hotmail.com

Ângela Maria Santiago

ORCID: https://orcid.org/0000-0001-7108-6890

Universidade Estadual da Paraíba, Brasil

E-mail: angelamariasantiago01@gmail.com

Pablícia Oliveira Galdino

ORCID: https://orcid.org/0000-0001-6996-0550 Universidade Estadual da Paraíba, Brasil E-mail: pabliciaog@hotmail.com

Mércia Melo de Almeida Mota

ORCID: https://orcid.org/0000-0002-1336-9355

Universidade Federal de Campina Grande, Brasil E-mail: mercia01@gmail.com

\begin{abstract}
Resumo
O presente estudo teve como objetivo extrair amido de sementes de feijão preto e ajustar modelos matemáticos empíricos aos dados obtidos experimentalmente na sua cinética de secagem. A extração do amido das sementes de feijão preto foi realizada fazendo-se o uso de uma solução de metabissulfito de sódio $(0,5 \%)$. A pasta obtida após o processo de extração foi submetida a uma cinética de secagem nas temperaturas de 40,50 e $60{ }^{\circ} \mathrm{C}$ e velocidade do ar de $1,5 \mathrm{~m} \mathrm{~s}{ }^{-}$ 1. Aos dados experimentais foram ajustados modelos matemáticos empíricos, no qual foram avaliados através dos indicadores estatísticos coeficiente de determinação $\left(\mathrm{R}^{2}\right)$ e função qui-quadrado $\left(\chi^{2}\right)$ e por fim o rendimento de extração foi calculado como porcentagem de massa do amido obtido em relação à massa bruta das sementes de feijão preto. O coeficiente de determinação $\left(\mathrm{R}^{2}\right)$ de todos os modelos aplicados apresentaram valores superiores a 0,98 $\left(\mathrm{R}^{2}>0,98\right)$ e o modelo de Page apresentou os menores valores para função qui-quadrado, no qual variam de 0,005637 a 0,003212. Os valores de rendimento de extração variaram de 45,35 a 47,62\% sendo o maior percentual obtido na temperatura de $60{ }^{\circ} \mathrm{C}$. No entanto, o modelo de Page na temperatura de $50{ }^{\circ} \mathrm{C}$ é o mais adequando para representar esse estudo, pois apresentou o maior valor de $\mathrm{R}^{2}(0,9998)$ e o menor valor da função qui-quadrado $\left(0,3212 \times 10^{-2}\right)$.
\end{abstract}


Palavras-chave: Água livre; Carboidrato; Conservação; Extração; Page.

\begin{abstract}
The present study aimed to extract starch from black bean seeds and adjust empirical mathematical models to the data obtained experimentally in its drying kinetics. Starch was extracted from black bean seeds using a sodium metabisulfite solution $(0.5 \%)$. The paste obtained after the extraction process was subjected to drying kinetics at temperatures of 40 , 50 and $60{ }^{\circ} \mathrm{C}$ and an air speed of $1.5 \mathrm{~m} \mathrm{~s}^{-1}$. Empirical mathematical models were adjusted to the experimental data, in which the coefficient of determination $\left(\mathrm{R}^{2}\right)$ and chi-square function $\left(\chi^{2}\right)$ were evaluated using the statistical indicators and finally the extraction yield was calculated as a percentage of the starch mass obtained in relation to the mass of black beans. The determination coefficient $\left(R^{2}\right)$ of all applied models showed values greater than $0.98\left(R^{2}>0.98\right)$ and the Page model showed the lowest values for the chi-square function, in which they vary from 0.005637 to 0,003212 . The extraction yield values varied from 45.35 to $47.62 \%$, the highest percentage being obtained at a temperature of 60 ${ }^{\circ} \mathrm{C}$. However, the Page model at a temperature of $50^{\circ} \mathrm{C}$ is the most adequate to represent this study, as it presented the highest value of R2 (0.9998) and the lowest value of the chi-square function $(0.3212 \times 10-2)$.
\end{abstract}

Keywords: Free water; Carbohydrate; Conservation; Extraction; Page.

\title{
Resumen
}

El presente estudio tuvo como objetivo extraer almidón de semillas de frijol negro y ajustar modelos matemáticos empíricos a los datos obtenidos experimentalmente en su cinética de secado. El almidón se extrajo de las semillas de frijol negro usando una solución de metabisulfito de sodio $(0,5 \%)$. La pasta obtenida tras el proceso de extracción se sometió a cinética de secado a temperaturas de 40,50 y $60^{\circ} \mathrm{C}$ y una velocidad del aire de $1,5 \mathrm{~m} \mathrm{~s}^{-1}$. Se ajustaron modelos matemáticos empíricos a los datos experimentales, en los cuales se evaluó el coeficiente de determinación $\left(\mathrm{R}^{2}\right)$ y la función chi-cuadrado $\left(\chi^{2}\right)$ mediante los indicadores estadísticos, y finalmente se calculó el rendimiento de extracción como porcentaje de la masa de almidón obtenida en relación a la masa de frijoles negros. El coeficiente de determinación $\left(\mathrm{R}^{2}\right)$ de todos los modelos aplicados mostró valores mayores a $0.98\left(\mathrm{R}^{2}>0.98\right)$ y el modelo de Page mostró los valores más bajos para la función chi-cuadrado, en la cual varían de 0.005637 a 0,003212. Los valores de rendimiento de extracción variaron de 45,35 a $47,62 \%$, obteniéndose el porcentaje más alto a una temperatura de $60{ }^{\circ} \mathrm{C}$. Sin embargo, el modelo de Page a una temperatura de $50^{\circ} \mathrm{C}$ es el más adecuado para representar este estudio, ya que presentó el valor más alto de R2 (0.9998) y el valor más bajo de la función chi-cuadrado (0.3212x10-2).

Palabras clave: Agua libre; Carbohidrato; Conservación; Extracción; Page.

\section{Introdução}

Estudos têm mostrado que o feijão preto (Phaseolus vulgaris L.) é uma leguminosa que contém muitos componentes benéfico para o corpo humano, incluindo lipídios, vitaminas, celulose e proteínas (Frota et al., 2010; Barros et al., 2018; Li et al., 2020). Segundo Rosa-Millán et al. (2019), o feijão é uma fonte adequada de carboidratos complexos, em que o amido é a fração predominante (50-60 g/100 g). O amido é um dos componentes mais importantes dos sistemas alimentares, pois o mesmo serve como uma fonte de energia na dieta humana (Fabbri et al., 2016; Chi et al., 2020).

No entanto, o amido quando é extraído apresenta uma elevada atividade de água, necessitando que seja aplicada alguma técnica de conservação, visando sua obtenção na forma de pó e consequentemente garantindo uma maior estabilidade durante o seu armazenamento. E uma dessas técnicas é a secagem convectiva, que segundo Santos et al. (2019a) e Almeida et al. (2020a) este processo envolve a redução do teor de água do produto até que o mesmo atinja um nível seguro, podendo ser aplicada para garantir a preservação da qualidade fisiológica e físico-química do produto a ser armazenado durante um longo período de tempo.

Nos estudos relacionados aos sistemas de secagem, o dimensionamento, a otimização e a determinação da viabilidade de sua aplicação comercial podem ser feitos através de simulações matemáticas, em que para isso faz-se uso de modelos matemáticos que possam representar satisfatoriamente a perda de água durante o processo de secagem (Araújo et al., 2017). Nesse contexto, o presente estudo tem como objetivo extrair amido de sementes de feijão preto e ajustar modelos matemáticos empíricos aos dados obtidos experimentalmente na sua cinética de secagem.

\section{Metodologia}

O feijão preto (Phaseolus vulgaris L.) foi adquirido no comércio local da cidade de Natal-RN. 


\subsection{Extração do amido}

Inicialmente para extração do amido as sementes de feijão preto foram trituradas e imersas em uma solução de metabissulfito de sódio $(0,5 \%)$ na proporção de $1: 2(\mathrm{~m} / \mathrm{v})$ por 72 horas, seguindo o procedimento proposto por Bento et al. (2019). As suspensões foram filtradas, lavadas com água destilada e decantadas por um período de 24 horas por 5 vezes, em ambiente refrigerado a $7{ }^{\circ} \mathrm{C}$ para evitar ação enzimática ou fermentativa durante o processo de decantação. O sobrenadante foi descartado e o decantado foi submetido a secagem.

\subsection{Cinética de secagem}

A secagem das pastas de amido de feijão preto foram realizadas em triplicata, utilizando uma estufa de secagem com circulação de ar forçado ajustado para operar nas temperaturas de 40,50 e $60{ }^{\circ} \mathrm{C}$ e com velocidade de ar de $1,5 \mathrm{~m} \mathrm{~s}^{-1}$, nas quais as amostras foram distribuídas uniformemente em bandejas de aço inoxidável, formando uma camada fina. Os dados experimentais da perda de água ao longo do processo de secagem foi expresso como a razão de umidade (X*), conforme apresentado na (Equação 1).

$$
X^{*}=\frac{X(t)-X_{e q}}{X_{i}-X_{e q}}
$$

Onde: $\mathrm{X}^{*}$ é a razão de umidade (adimensional), Xeq é o equilíbrio teor de umidade (base seca), $\mathrm{X}(\mathrm{t})$ é o teor de umidade real da amostra no momento t (base seca) e Xi é a umidade inicial conteúdo (base seca).

Quatro funções empíricas Handerson \& Pabis (Equação 2), Page (Equação 3), Lewis (Equação 4) e Silva et alii (Equação 5) foram ajustadas aos conjuntos de dados experimentais por regressão não linear com o software LAB Fit (Silva \& Silva, 2008).

$$
\begin{aligned}
& X^{*}=e^{-a t} \\
& X^{*}=e^{-a t b} \\
& X^{*}=a e^{-b t} \\
& X^{*}=e^{-a t-b \sqrt{t}}
\end{aligned}
$$

Onde: $\mathrm{X}^{*}$ é a razão de umidade (adimensional), "a" e "b" são os parâmetros dos modelos e t é o tempo de secagem.

Os resultados dos modelos empíricos foram avaliados e comparados usando o qui-quadrado $\left(\chi^{2}\right)$ (Equação 6) e o coeficiente de determinação R2.

$$
X^{2}=\frac{\sum_{i=1}^{N}\left(X_{\mathrm{exp}, i}^{*}-X_{p r e, i}^{*}\right)^{2}}{N-n}
$$


Onde: é a função qui-quadrado, é a razão de umidade experimental, é a razão de umidade simulada, $\mathrm{N}$ é o número de pontos experimentais e n é o número de coeficientes e constantes no modelo.

\subsection{Rendimento de extração}

O rendimento de extração foi calculado como porcentagem de massa do amido obtido em relação à massa bruta das sementes de feijão preto e expresso em base seca, por meio da Equação 7.

$$
R=\frac{M s}{M r} \times 100
$$

Onde: Ms é a massa do amido em base seca (g); Mr é a massa do feijão preto (g).

\subsection{Análise estatística}

Os dados experimentais obtidos serão analisados em triplicata e os resultados submetidos à análise de variância de fator único (ANOVA) de 5\% de probabilidade. Para o desenvolvimento das análises estatísticas será utilizado o software Assistat 7.7

\section{Resultados e Discussão}

Na Tabela 1 estão dispostos os resultados obtidos para os modelos empíricos aplicados a cinética de secagem da pasta de amido de feijão preto.

Tabela 1 - Valores obtidos pelos ajustes de modelos matemáticos aos dados experimentais da cinética de secagem da pasta de amido de feijão preto

\begin{tabular}{cccc}
\hline Modelo & $\mathrm{T}\left({ }^{\circ} \mathrm{C}\right)$ & $a$ & $b$ \\
\hline \multirow{3}{*}{ Handerson \& Pabis } & 40 & 1,0539 & $0,5100 \times 10^{-2}$ \\
& 50 & 1,0720 & $0,8554 \times 10^{-2}$ \\
& 60 & 1,0949 & $0,1634 \times 10^{-1}$ \\
\hline \multirow{2}{*}{ Page } & 40 & $0,6598 \times 10^{-3}$ & 1,3724 \\
& 50 & $0,9940 \times 10^{-3}$ & 1,4399 \\
& 60 & $0,3201 \times 10^{-2}$ & 1,8378 \\
\hline \multirow{2}{*}{ Lewis } & 40 & $0,4731 \times 10^{-2}$ & - \\
& 50 & $0,7684 \times 10^{-2}$ & - \\
\hline \multirow{2}{*}{ Silva et alii } & 60 & $0,1490 \times 10^{-1}$ & - \\
& 40 & $0,6459 \times 10^{-2}$ & $-0,2231 \times 10^{-1}$ \\
& 50 & $0,1146 \times 10^{-1}$ & $-0,3560 \times 10^{-1}$ \\
& 60 & $0,2189 \times 10^{-1}$ & $-0,4778 \times 10^{-1}$ \\
\hline
\end{tabular}

Fonte: Própria (2021)

Observa-se que os parâmetro " $a$ " e " $b$ " das equações empíricas aplicadas sofreram influência com a elevação da temperatura do ar de secagem de 40 para $60^{\circ} \mathrm{C}$. O parâmetro " $b$ " representa a constante da taxa de secagem nos modelos empregados, aumentando com a elevação da temperatura e representando os efeitos das condições externas de secagem (Santos 
et al., 2020). Esse mesmo comportamento também foi observado por Almeida et al. (2020b) ao aplicarem o processo de secagem em sementes de feijão azuki nas temperaturas variando de 40 a $60{ }^{\circ} \mathrm{C}$. Os valores obtidos para os indicadores estatísticos coeficiente de determinação $\left(\mathrm{R}^{2}\right)$ e função qui-quadrado $\left(\chi^{2}\right)$ estão apresentados na Tabela 2.

Tabela 2 - Coeficiente de determinação $\left(\mathrm{R}^{2}\right)$ e função qui-quadrado $\left(\chi^{2}\right)$ obtidos pelos ajustes de modelos matemáticos aos dados experimentais da cinética de secagem da pasta de amido de feijão preto

\begin{tabular}{cccc}
\hline Modelo & $\mathrm{T}\left({ }^{\circ} \mathrm{C}\right)$ & $R^{2}$ & $\chi^{2}$ \\
\hline \multirow{3}{*}{ Handerson \& Pabis } & 40 & 0,9902 & $0,4755 \times 10^{-1}$ \\
& 50 & 0,9887 & $0,4429 \times 10^{-1}$ \\
& 60 & 0,9870 & $0,3481 \times 10^{-1}$ \\
\hline \multirow{2}{*}{ Page } & 40 & 0,9989 & $0,5637 \times 10^{-2}$ \\
& $\mathbf{5 0}$ & $\mathbf{0 , 9 9 9 8}$ & $\mathbf{0 , 3 2 1 2 \times 1 0 ^ { - 2 }}$ \\
& 60 & 0,9994 & $0,4672 \times 10^{-2}$ \\
\hline \multirow{2}{*}{ Lewis } & 40 & 0,9917 & $0,6656 \times 10^{-1}$ \\
& 50 & 0,9898 & $0,6911 \times 10^{-1}$ \\
& 60 & 0,9879 & $0,4788 \times 10^{-1}$ \\
\hline \multirow{2}{*}{ Silva et alii } & 40 & 0,9945 & $0,2586 \times 10^{-1}$ \\
& 50 & 0,9949 & $0,2049 \times 10^{-1}$ \\
& 60 & 0,9931 & $0,1750 \times 10^{-1}$ \\
\hline
\end{tabular}

Fonte: Própria (2021).

Analisando a Tabela 2, o coeficiente de determinação $\left(\mathrm{R}^{2}\right)$ de todos os modelos aplicados apresentaram valores superiores a 0,98 $\left(\mathrm{R}^{2}>0,98\right)$ para todas as temperaturas aplicadas. Mas, a avaliação de apenas um único parâmetro estatístico não constitui um bom critério de seleção de modelos não-lineares. Sendo, portanto, necessário a análise de outros parâmetros como a função qui-quadrado (Silva et al., 2019b; Santos et al., 2019b). Em análise do qui-quadrado ( $\chi^{2}$ ), pode-se observar que o modelo de Page têm os menores valores, no qual variam de 0,005637 a 0,003212 quando a temperatura do ar de secagem variou de 40 a $60{ }^{\circ} \mathrm{C}$. Dessa forma o modelo de Page na temperatura de $50{ }^{\circ} \mathrm{C}$ é o mais adequando para esse estudo, pois apresentou o maior valor de $\mathrm{R}^{2}(0,9998)$ e o menor valor da função qui-quadrado $\left(0,3212 \times 10^{-2}\right)$.

Na Figura 1, pode-se observar o modelo Page como o que melhor descreve a cinética de secagem da pasta de amido de feijão preto para as temperaturas de secagem aplicadas. 
Figura 1 - Cinética de secagem da pasta de amido de feijão preto, simulada pelo modelo matemático de Page

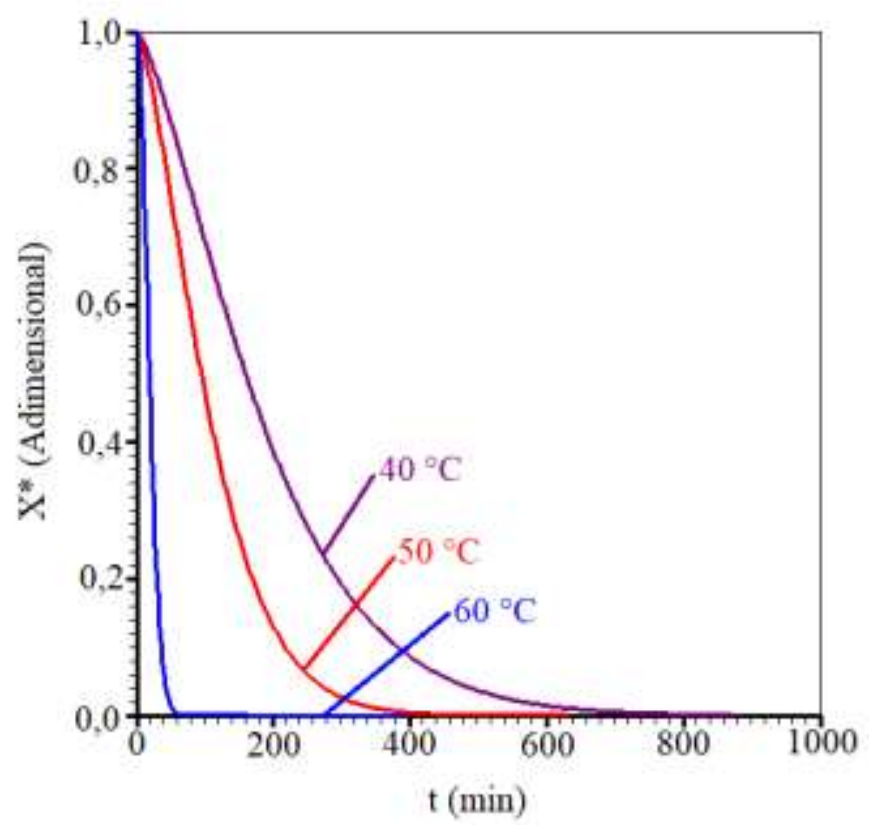

Fonte: Própria (2021)

O aumento da temperatura do ar de secagem reduziu o tempo de secagem das pastas de amido, cujos tempos foram de 870,630 e $390 \mathrm{~min}$ para as temperaturas de 40,50 e $60^{\circ} \mathrm{C}$, respectivamente. Essa redução do tempo de secagem está relacionada ao aumento da taxa de perda, visto que em maiores temperaturas as moléculas de água sofrem uma menor resistência, ou seja, fazendo com que a água seja menos ligada ás moléculas que constituem a massa seca do material.

Após o processo de secagem das pastas de amido de feijão preto nas respectivas temperaturas, a Tabela 3 , apresenta os valores calculados para o rendimento de extração.

Tabela 3 - Rendimento de extração de amido de feijão preto para cada temperatura de secagem

\begin{tabular}{cc}
\hline $\mathrm{T}\left({ }^{\circ} \mathrm{C}\right)$ & Rendimento $(\%)$ \\
\hline 40 & $45,35^{\mathrm{a}} \pm 0,55$ \\
50 & $46,72^{\mathrm{a}} \pm 0,98$ \\
60 & $47,62^{\mathrm{a}} \pm 1,12$ \\
\hline
\end{tabular}

Fonte: Própria (2021)

Observa-se que os valores de rendimento de extração variaram de 45, 35 a 47,62\% sendo o maior percentual obtido na temperatura de $60{ }^{\circ} \mathrm{C}$, no entanto, estatisticamente os valores médios obtidos não apresentaram diferenças significativas ao nível de $5 \%$ de probabilidade.

\section{Conclusão}

O modelo matemático de Page na temperatura de $50{ }^{\circ} \mathrm{C}$, apresentou-se como o de melhor ajuste aos dados experimentais da cinética de secagem das pastas de amido de feijão preto, pois apresentou o maior valor de $\mathrm{R}^{2}$ e o menor valor da função qui- 
quadrado. O menor tempo de secagem foi obtido para a temperatura de $60{ }^{\circ} \mathrm{C}(390 \mathrm{~min})$ em decorrência do maior gradiente de temperatura. E por fim, o rendimento de extração foi superior a $40 \%$ para as três temperaturas de aplicadas, no entanto, não apresentaram diferenças estatísticas. Como sugestão de trabalhos futuros, pode-se realizar um caracterização física, físicoquímica e tecnológica do amido em pó.

\section{Referências}

Almeida, R. L. J., Santos, N. C., dos Santos Pereira, T., de Queiroga, A. P. R., de Alcântara Silva, V. M., de Alcântara Ribeiro, V. H., ... \& Borges, E. M. E. S. (2020b). Cinética de secagem do feijão azuki: modelagem matemática e propriedades termodinâmicas. Research, Society and Development, 9(3), 3.

Almeida, R. L., Santos, N. C., dos Santos Pereira, T., de Alcântara Silva, V. M., Cabral, M. B., Barros, E. R., ... \& da Silva, L. R. I. (2020a). Determinação de compostos bioativos e composição físico-química da farinha da casca de jabuticaba obtida por secagem convectiva e liofilização. Research, Society and Development, 9(1), e157911876-e157911876.

Araujo, W. D., Goneli, A. L. D., Corrêa, P. C., Hartmann Filho, C. P., \& Martins, E. A. S. (2017). Modelagem matemática da secagem dos frutos de amendoim em camada delgada. Revista Ciência Agronômica, 48(3), 448-457.

Barros, L. F. T. D., Escobar, T. D., Ribeiro, P. F. D. A., \& Kaminski, T. A. (2018). Muffins adicionados de farinha de feijão de diferentes classes. Brazilian Journal of Food Technology, 21.

Bento, J. A. C., Ferreira, K. C., de Oliveira, A. L. M., Lião, L. M., Caliari, M., \& Júnior, M. S. S. (2019). Extraction, characterization and technological properties of white garland-lily starch. International journal of biological macromolecules, 135, 422-428.

Chi, C., Li, X., Zhang, Y., Chen, L., Li, L., \& Miao, S. (2020). Progress in tailoring starch intrinsic structures to improve its nutritional value. Food Hydrocolloids, 106447.

Fabbri, A. D., Schacht, R. W., \& Crosby, G. A. (2016). Evaluation of resistant starch content of cooked black beans, pinto be ans, and chickpeas. NFS journal, 3, $8-12$.

Frota, K. D. M. G., Morgano, M. A., Silva, M. G. D., Araújo, M. A. D. M., \& Moreira-Araújo, R. S. D. R. (2010). Utilização da farinha de feijão-caupi (Vigna unguiculata L. Walp) na elaboração de produtos de panificação. Food Science and Technology, 30, 44-50.

Li, P., Li, Y., Wang, L., Zhang, H., Qi, X., \& Qian, H. (2020). Study on water absorption kinetics of black beans during soaking. Journal of Food Engineering, 110030.

Rosa-Millán, J.D.L., Heredia-Olea, E., Perez-Carrillo, E., Guajardo-Flores, D., \& Serna-Saldívar, S. R. O. (2019). Effect of decortication, germination and extrusion on physicochemical and in vitro protein and starch digestion characteristics of black beans (Phaseolus vulgaris L.). LWT, $102,330-337$.

Santos, N. C., Silva, V. M. A., Ribeiro, V. H. A., Barros, S. L., Almeida, R. L. J., Luiz, M. R., \& Beserra, Y. A. S. (2019b). Secagem convectiva de cascas de kiwi cv. hayward, difusividade efetiva e energia de ativação. In: Paulo Roberto Megna Francisco; George do Nascimento Ribeiro; Paulo da Costa Medeiros. (Org.). Caderno de Pesquisa, Ciência e Inovação. 3ed.Campina Grande: EPGRAF, 2, 82-89.

Santos, N. C., Silva, W. P., Barros, S. L., Araújo, A. J. B., Gomes, J. P., Almeida, R. L. J., Nascimento, A. P. S., Almeida, R. D., Silva, C. M. D. P. S., Queiroz, A. J. M., \& Figueirêdo, R. M. F. (2019a). Study on drying of black rice (Oryza sativa L.) grains: physical-chemical and bioactive quality. Journal of Agricultural Science, 11(9), 203-212.

Santos, N. C., da Silva, W. P., Barros, S. L., Almeida, R. L. J., de Brito Araújo, A. J., \& da Silva Nascimento, A. P. (2020). Red rice (Oryza sativa L.) use in flour production: Convective drying and bioactive quality. Journal of Food Process Engineering, 43(10), e13490.

Silva, E. C. O., da Silva, W. P., Gomes, J. P., Silva, C. M. D. P. S., Alexandre, H. V., Farias, V. S. O., ... \& de Figuiredo, R. M. F. (2019b). Drying of Albedo and Whole Peel of Yellow Passion Fruit. Journal of Agricultural Science, 11(6). 